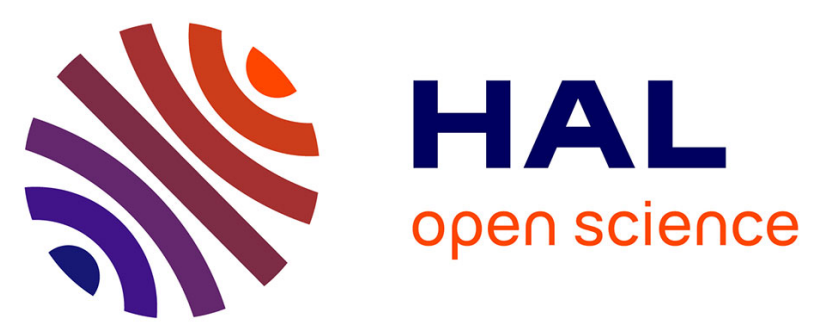

\title{
Influence of tumor-associated macrophages and HLA class I expression according to HPV status in head and neck cancer patients receiving chemo/bioradiotherapy
}

Dan Ou, Julien Adam, Ingrid Garberis, Pierre Blanchard, France Nguyen, Antonin Levy, Odile Casiraghi, Philippe Gorphe, Ingrid Breuskin, François Janot, et al.

\section{To cite this version:}

Dan Ou, Julien Adam, Ingrid Garberis, Pierre Blanchard, France Nguyen, et al.. Influence of tumorassociated macrophages and HLA class I expression according to HPV status in head and neck cancer patients receiving chemo/bioradiotherapy. Radiotherapy \& Oncology, 2019, 130, pp.89 - 96. 10.1016/j.radonc.2018.08.013 . hal-03484538

\author{
HAL Id: hal-03484538 \\ https://hal.science/hal-03484538
}

Submitted on 20 Dec 2021

HAL is a multi-disciplinary open access archive for the deposit and dissemination of scientific research documents, whether they are published or not. The documents may come from teaching and research institutions in France or abroad, or from public or private research centers.
L'archive ouverte pluridisciplinaire HAL, est destinée au dépôt et à la diffusion de documents scientifiques de niveau recherche, publiés ou non, émanant des établissements d'enseignement et de recherche français ou étrangers, des laboratoires publics ou privés.

\section{(ㄷ)(1) $\$$}

Distributed under a Creative Commons Attribution - NonCommerciall 4.0 International 


\section{Influence of tumor-associated macrophages and HLA class I expression according to HPV status in head and neck cancer patients receiving chemo/bioradiotherapy}

Dan $\mathrm{Ou}^{1,2,3,4,5}$, Julien Adam ${ }^{6}$, Ingrid Garberis ${ }^{6,7}$, Pierre Blanchard ${ }^{1}$, France Nguyen ${ }^{1}$, Antonin Levy ${ }^{1,2,3}$, Odile Casiraghi ${ }^{6}$, Philippe Gorphe ${ }^{8}$, Ingrid Breuskin ${ }^{8}$, François

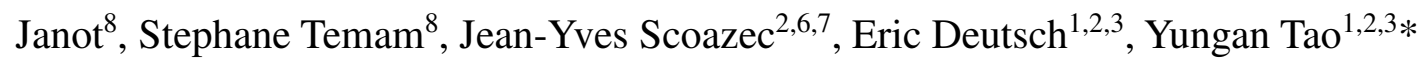

1 Department of Radiation Oncology, Gustave Roussy Cancer Campus, Villejuif, France

2 Université Paris Sud, France

3 INSERM1030 molecular radiotherapy, Villejuif, France

4 Department of Radiation Oncology, Fudan University Shanghai Cancer Center, Shanghai, China

5 Department of Oncology, Shanghai Medical College, Fudan University, Shanghai, China

6 Department of Pathology, Gustave Roussy Cancer Campus, Villejuif, France

7 INSERM US23/CNRS UMS3655, Molecular analysis, modelling and imaging of cancer disease, Experimental and Translational Pathology, Villejuif, France

8 Department of Head and Neck Oncology, Gustave Roussy Cancer Campus, Villejuif, France

* Corresponding author: Yungan Tao MD, PhD, Department of Radiation Oncology, Gustave Roussy Cancer Campus, 114 Rue Edouard Vaillant, 94805 Villejuif France.

Email: yungan.tao@gustaveroussy.fr

Tel: 0033142116532

Fax: 0033142115253 
No. of pages: 23

Number of figures: 3

Number of tables: 3

Running head: Tumor-associated macrophages and p16 in HNSCC

Keywords: Head and neck cancers; Human papillomavirus; tumor-associated macrophage; cetuximab; chemoradiotherapy; HLA class I 


\title{
Practical contouring guidelines with an MR-based atlas of brainstem structures involved in radiation-induced nausea and vomiting
}

\author{
A Beddok $^{1,7}$, J-C. Faivre ${ }^{2}$, A Coutte ${ }^{1}$, J Le Guévelou ${ }^{3}$, J Welmant $^{4}$, J-B Clavier ${ }^{5}$, S Guihard ${ }^{5}$, G

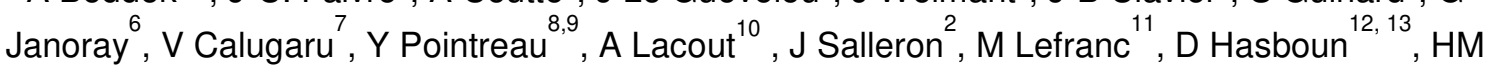 \\ Duvernoy $^{14}$, J Thariat $^{3,15}$
}

1. Department of Radiation Oncology. University hospital of Amiens, Picardie, 80054 Amiens, France

2. Lorraine Institute of Cancerology - Alexis-Vautrin Comprehensive Cancer Center, Academic Radiation Oncology \& Brachytherapy Department, 6 avenue de Bourgogne, Vandœuvre-lès-Nancy; F-54519, France

3. Department of Radiation Oncology. Centre François Baclesse, 3 Av General Harris, 14000 Caen, France

4. Department of Radiation Oncology. Montpellier Institute of Cancer, 208 Avenue des Apothicaires, 34298 Montpellier, France

5. Department of Radiation Oncology. Strasbourg Insitute of Cancerology - Paul Strauss Cancer Center, 3 rue de la Porte de l'Hôpital - BP 42 - 67065 Strasbourg Cedex, France

6. Department of Radiation Oncology. Tours Regional University Center, 2 Boulevard Tonnellé, 37000 Tours, France

7. Department of Radiation Oncology. Curie Institute, 26 rue d'Ulm, 75005 Paris, France

8. Jean Bernard Radiation Oncology Center, 9 Rue Beauverger, 72000 Le Mans, France

9. CORad Department, Henry S Kaplan - Bretonneau Regional University Hospital Center, 2 Bd Tonnellé, 37044 Tours, France ; CNRS, UMR 7292 "Génétique, Immunothérapie, Chimie et Cancer", Tours, France

10. Department of Radiology. Centre médico -chirurgical - ELSAN, 83 avenue Charles de Gaulle, 15000 AURILLAC France

11. Department of Neurosurgery. University hospital of Amiens, Picardie, 80054 Amiens, France

12. Department of Neuroradiology. Pitié-Salpêtrière University Hospital. 91 boulevard de l'hôpital, Paris, France.

13. Department of Neuroanatomy. Pitié-Salpêtrière University Hospital. 91 boulevard de l'hôpital, Paris, France.

14. Besançon Regional University Hospital Center, laboratoire de pathology, Centre Hospitalier Régional Universitaire de Besançon (Besançon), 2, place Saint-Jacques, 25030 Besançon cedex, France

15. Laboratoire de physique corpusculaire IN2P3/ENSICAEN - UMR6534 - Unicaen - Normandie Université Boulevard du Marechal Juin 14050 Caen, France

* Corresponding author: Arnaud Beddok, 11 rue de la Glacière, 75013 Paris, France

a.beddok@gmail.com, (33) (0)6 19174502

Number of pages: 9

Number of tables and figures: 6

Number of supplemental data: 1

Short title: Atlas of brainstem structures involved in RINV

Keywords: nausea, vomiting, brainstem, area postrema, radiotherapy 


\section{Abstract}

Background and Purpose: The objective of this project was to define consensus guidelines for delineating brainstem substructures (dorsal vagal complex, including the area postrema) involved in radiation-induced nausea and vomiting (RINV). The three parts of the brainstem are rarely delineated, so this study was also an opportunity to find a consensus on this subject.

Materials and Methods: The dorsal vagal complex (DVC) was identified on autopsy sections and endoscopic descriptions. Anatomic landmarks and boundaries were used to establish radio-anatomic correlations on CT and Magnetic Resonance Imaging (MRI). Additionally, delineation of RINV structures was performed on MRI images and reported on CT scans. Next, guidelines were provided to eight radiation oncologists for delineation guidance of these RINV-related structures on DICOM-RT images of two patients being treated for a nasopharyngeal carcinoma. Interobserver variability was computed.

Results: The DVC and the three parts of the brainstem were defined with a concise description of their main anatomic boundaries. The interobserver analysis showed that the DVC, the midbrain, the pons, and the medulla oblongata delineations were reproducible with $\mathrm{KI}=0.72,0.84,0.94$ and 0.89 , respectively. The Supplemental Material section provides an atlas of the consensus guidelines projected on 1-mm MR axial slices.

Conclusions: This RINV-atlas was feasible and reproducible for the delineation of RINV structures on planning CT using fused MRI. It may be used to prospectively assess dose-volume relationship for RINV structures and occurrence of nausea vomiting during intracranial or head and neck irradiation. 


\section{Introduction}

Intensity-modulated radiation therapy (IMRT) leads to increased reduction in late toxicities compared to 3-dimensional conformal radiation therapy (3D-CRT) techniques (1). In IMRT, dose distributions conform to tumor topography while simultaneously limiting the normal tissue volume exposed to relatively high radiation doses. Therefore, IMRT has become the recommended treatment strategy for many cases of locally advanced head and neck cancer (HNC). However, IMRT treatment plans for HNC typically use a high number of radiation fields and may be associated with new beam-path nontarget tissue toxicities such as anterior oral mucositis, occipital scalp hair loss, headache, nausea, and vomiting (2). Radiation-induced nausea and vomiting (RINV) can occur during the irradiation of a small part of the brainstem called the dorsal vagal complex (DVC) (3). This structure of the medulla oblongata includes the area postrema (AP), the nucleus of the solitary tract (NST), and the dorsal motor nucleus of the vagus (DMV) (4). Several retrospective studies have recently demonstrated a statistical correlation between the grade of RINV and the dose in the DVC (5-8). In most of these studies, the location of AP and other structures implicated in RINV were determined on CT scan or MRI by a radiation oncologist with the help of an expert neuroradiologist. However, it remained difficult to accurately identify the true boundaries of these small structures using standard imaging techniques (9). In the present study, we propose an atlas of RINV-related organs based on pathologic, anatomic, and endoscopic descriptions of the fourth ventricle floor (10-16). This atlas was validated as a consensus by an interobserver analysis which compared the delineation of eight different radiation oncologists.

\section{Methods}

\section{Population: working group}

A multidisciplinary working group (WG) was created to develop these new recommendations for central nervous system (CNS) substructures involved in RINV. All physicians who contributed to the creation of these guidelines are listed as co-authors of this manuscript. The general principles guiding the activities of the WG were: (1) to integrate as accurately as possible anatomic knowledge and previously defined surgical and radiological guidelines into a set of recommendations based on axial CT and MR sections, and (2) to minimize variations in interpretation of the guidelines between oncologists by refining the description of the boundaries at various locations.

\section{Construction of the atlas}

A first tutorial was created to help the WG delineate the DVC. We assumed a lack of anatomic knowledge on the part of the radiation oncologists regarding this small structure which is not visible on CT and MR images. This tutorial was created using MRI and CT-scan images of a patient treated with 
stereotactic radiotherapy for a pituitary adenoma. CT images were obtained using a dosimetric CT with a slice thickness of $0.625 \mathrm{~mm}$. MR images were obtained using a $1.5 \mathrm{~T}$ magnet. The patient was scanned in the same position as the dosimetric CT, with a slice thickness of $0.5 \mathrm{~mm}$. An image fusion between CT and MR images was performed with studies of pathologic and anatomic surgical descriptions of the brainstem and floor of the fourth ventricle (10-16). The location, size, and true boundaries of the DVC were then determined with the help of three experts in brainstem anatomy ( $\mathrm{Pr}$ H.M. Duvernoy, Dr D. Hasboun, Dr M. Lefranc). The three parts of the brainstem are rarely delimited, so this study was also an opportunity to find a consensus on this subject.

Secondly, each member of the working group delineated the DVC and the three parts of the brainstem in a CT set from one patient treated for nasopharyngeal neoplasm (patient A). The CT images (1-mm slice thickness) were carried out with patient in a supine position on a multidetector-row spiral CT scanner. A fusion between MR and CT images was necessary to determine the location of the DVC. The contours of all radiation oncologists were compared (see the method of comparison below). Subsequently, the outcome of this procedure was individually presented to and discussed with each observer in order to identify the most frequent inconsistencies and to formulate consensus guidelines.

Finally, consensus delineations were depicted on axial T2-weighted MRI in an atlas of structures involved in RINV with a 1-mm slice thickness (atlas in supplementary data). A second inter-observer analysis was performed to confirm validity and reproducibility of this atlas. In this second analysis, each member of the working group delineated the DVC and the three parts of the brainstem in a CT set from the same patient $A$ and from another patient $B$.

\section{Methods of comparison}

These methods were used for the first (Time 1) and the second (Time 2) interobserver analysis. The evaluation of the variability of contours was benchmarked using a "reference" contour (delineated with the help of the three experts of the brainstem: HMD, DH, and ML). ARTISTRUCT'M software version 1.0 (AQUILAB®, Lille, France) was used to evaluate and compare the contours, both qualitatively and quantitatively. For the first step, each volume submitted by each of the eight radiation oncologists in the WG (DVC, midbrain, pons, and medulla oblongata) was compared with the reference volume proposed by the three experts. In the second step, different indices reflecting the correlation of the volume with the "experts reference contour" for all volumes were calculated. Three of these indices are based on volume ratios: the Volume Ratio (VR) between the volume of one of the eight radiation oncologists and the volume of the experts, the Common Delineated Volume (CDV), and the Additional Delineated Volume (ADV). Then, the kappa index (KI) and the Overlap Volume (OV) were calculated. Patient A's indices of the Time 1 were compared with Patient A's indices of the Time 2 (17). Another patient (Patient B) allowed us to confirm the validity of the second tutorial as an atlas for the delineation of the DVC. The delineation of the three parts of the brainstem was also analyzed and reported for Patient B. 


\section{Statistical analysis}

Delineation evaluation was conducted prospectively and comparatively. Statistical analysis was performed with R-Studio software (GNU Affero General Public License v3 2007). Comparison between the volume obtained (eight radiation oncologists of the WG) and the experts' volume was done with the Student t-test. Comparative analysis of Patient A's indices of the Time 1 and Patient A's indices of the Time 2 after was done with the Student t-test for paired samples. Values of two-sided $p$ $<0.05$ were considered statistically significant.

\section{Results}

After the working group (WG) delineated the DVC and the three parts of the brainstem in the patient A, a first interobserver analysis (Time 1) was performed and the variability in delineation was discussed. Subsequently, the WG agreed on consensus definitions for these structures and formulated the final consensus guidelines for these structures implicated in RINV (available in supplemental data). A second interobserver analysis (Time 2) confirmed that the delineation of the DVC and the three parts of the brainstem was reproducible. In the following sections, each of these structures will be briefly reviewed with a short description of their main anatomic boundaries. The boundaries correspond to a patient lying supine with his head in a "neutral" position. The boundaries are summarized in Table 1.

\section{Midbrain}

The midbrain is the most cranial structure of the brainstem. The cranial limit is the cranial border of the cerebral aqueduct. This limit is clearly visible in the sagittal plane of the brainstem in MRI. The caudal limit is below the inferior coliculli. The midbrain is also limited anteriorly by the interpeduncular cistern and posteriorly by the quadrigeminal cistern.

\section{Pons}

The pons is similar to a bridge connecting the right and left cerebellar hemispheres. The cranial limit (which is also the caudal limit of the midbrain) is above the inferior coliculli of the midbrain. Posteriorly, the pons is limited by the floor of the fourth ventricle. Convincing arguments have led us to include the superior, middle, and inferior cerebellar peduncles in the pons structure because the dose constraints are the same as those for the pons.

\section{Medulla oblongata}

The medulla oblongata connects the spinal cord to the pons. The caudal limit of the medulla oblongata is located at the level of the foramen magnum (clearly visible on a sagittal view of the brainstem on a 
CT image). The superior half of the posterior surface of the medulla oblongata is the floor of the fourth ventricle, above the median aperture. This surface is characterized by a median sulcus, clearly visible on CT and MRI. Under the median aperture, which is also the cranial limit of the central canal, this median sulcus is not visible. The posterior border of the medulla is then the posterior cerebellomedullary cistern, whereas the lateral borders are limited by the lateral apertures and the lateral cerebello-medullary cisterns.

\section{Dorsal vagal complex (DVC)}

The dorsal vagal complex (DVC), consisting of the AP and the parts of the NST and the DMV connected to the AP, is located in the dorsal brainstem, in the floor of the fourth ventricle. The DVC integrates sensory and motor information of neurovegetative functions, including vomiting. In a posterior view, the floor of the fourth ventricle appears rhomboidal (Fig 1). The back surface of the pons and the superior half of the dorsal surface of the medulla oblongata form the floor of the fourth ventricle. Its superior angle is continuous with the cerebral aqueduct, while its inferior angle is continuous with the central canal. The floor is grooved vertically by a median sulcus. The area postrema (AP) is attached to the inferior angle of the floor of the fourth ventricle, overlying the lower portion of the vagal trigone and facing the Magendie foramen. In an endoscopic view, the AP is directly visible from the back through the median aperture (foramen of Magendie) and is joined by the opening of the central canal in the fourth ventricle (14). The AP has the appearance of arched wings opened on either side of the floor of the fourth ventricle, facing the Magendie foramen, and diverging upward from the central canal. The caudal limit of the DVC is easily determined on a sagittal view of the brainstem and must be delineated in the transversal plane of the obex, which is also in the plane of the central canal aperture (Fig. 1A, 1B) (16). This limit is a unique volume located at a distance of $52 \pm 3.5 \mathrm{~mm}$ from a line between the head and the body of the corpus callosum (Fig. 1C, 1D). The DVC is located on both sides of the median sulcus of the medulla oblongata (Fig $2 \mathrm{~A}, 2 \mathrm{~B}$ ). In order to be more reproducible, we proposed delineating a 4-mm diameter circle which included the different structures of the DVC (Fig. 2C). The cranio-caudal length of the AP is $5 \mathrm{~mm}(10)$.

Tables 2 and 3 show the results of the first and second interobserver analyses.

\section{Volume comparison}

In the first analysis (Time 1), the DVC delineated by the experts for the patient A was measured at $0.04 \mathrm{~cm}^{3}$ and extended $0.4 \mathrm{~cm}$ in the vertical plane. The average volume and height of the DVC delineated by the working group (WG) for the same patient $A$ were significantly different from that of the experts with a result of $0.14 \mathrm{~cm}^{3}(p=0.02)$ and $0.64 \mathrm{~cm}(p=0.008)$, respectively. In the second analysis (Time 2), the volume of Patient A's DVC delineated by the expert was $0.14 \mathrm{~cm}^{3}$ and extended $0.5 \mathrm{~cm}$ in the vertical plane. The average volume and height of Patient A's DVC delineated by the WG were not significantly different from that of the expert with a result of $0.14 \mathrm{~cm}^{3}(p$ 
$=0.52)$ and $0.5 \mathrm{~cm}(p=1)$, respectively. The analysis of the patient B confirmed that the DVC volume and height of the WG did not differ from the expert. The patient $B$ also allowed us to validate the reproducibility of the midbrain, the pons, and the medulla oblongata delineations (Table 3).

\section{Indices comparison}

In the first analysis (Time 1), the different indices obtained by the WG compared to the expert for the patient A were 3.29 for the Volume Ratio (VR), 74\% for the Common Delineated Volume (CDV), 72\% for the Additional Delineated Volume (ADV), 0.26 for the Overlap Volume (OV), and 0.40 for the Kappa Index (KI). In the second analysis (Time 2), the different indices obtained by the WG compared to the expert for the same patient A were 1 for the VR, 67\% for CDV, 33\% for the ADV, 0.53 for the $\mathrm{OV}$, and 0.67 for the $\mathrm{KI}$. The results obtained in the second analysis were significantly better than these ones obtained in the first analysis (Table 2 shows the mean differences for each index). These results showed a significant improvement in the reproducibility of the DVC delineation and validated the second tutorial as an atlas. The indices obtained by the WG compared to the expert for patient $B$ are reported in Table 2 for the DVC and in Table 3 for the three parts of the brainstem.

\section{Discussion}

Radiation-induced nausea and vomiting (RINV) were rare side effects with 3-dimensional conformal radiation therapy (3D-CRT) techniques. RINV seems to be more frequent since the development of new technologies such as intensity-modulated radiation therapy (IMRT) for the treatment of locally advanced head and neck cancer (HNC). The RINV atlas described above has thus been designed to prospectively assess dose-volume effects for structures previously identified as being involved in RINV.

In 2008, Rosenthal et al observed in a post hoc analysis that the reported rates of emesis (4\%) and nausea $(4 \%)$ in patients treated with 3D-CRT were substantially lower than those observed in their IMRT patient series. In recursive partitioning analysis (RPA), nausea and emesis were associated with the reconstructed mean dose to the brainstem of $>36$ Gy (2). Other previous studies on stereotactic radiosurgery have shown that the dose to the area postrema (AP) in the brainstem correlated with nausea and vomiting and that limiting the dose to even lower levels could reduce the incidence of these toxicities (3). Therefore, the MDA team surmised that RINV may be related to the dose in the dorsal vagal complex (DVC) which includes the AP. Ciura et al. in 2011 showed a positive relationship analysis between severe (grade 3 or greater) toxicity (nausea and vomiting) and brainstem mean dose and AP mean dose on logistic regression ( $p \leq 0.002)$ (7). In another study, the same team demonstrated that a dose-response association was detectable for $\operatorname{AP}(p=0,015)$ and after multiple statistical analyses (RPA, multivariate analysis), V24 (percentage of volume receiving at least $24 \mathrm{~Gy}$ ) to the AP $<76 \%$ was proposed to decrease the risk of RINV (8). Moreover, several other retrospective studies have recently demonstrated a statistical correlation between frequency and grade of RINV and 
the dose in the DVC. In 2008, Monroe et al. observed that the median dose to the DVC was significantly higher in patients with grade 1-2 nausea than in those with no RINV. In univariate analysis, patients with no acute nausea had a median DVC dose of $6.5 \mathrm{~Gy}$, whereas, those with grade 1-2 nausea had a median DVC dose of 26.9 Gy $(p=0.04)(5)$. However, the relation between the frequency and the grade of RINV and the dose in the DVC has neither been demonstrated in a multivariate analysis nor in the nasopharyngeal cancer, Lee et al. demonstrated that the dose to the DVC was not predictive of RINV (18). The lack of evidence between RINV and dose in the DVC could be explained by the lack of consensus for the delineation of the DVC. An accurate delineation would allow more reproducible and real results. Thus, providing atlas-based guidelines for the delineation of RINV structures appears relevant to routine practice. Not only can it be used to further define dose volume and toxicity relationships that may be used for NTCP modeling, but it also can be used to apply new OAR constraints for treatment optimization when needed.

Despite high resolution and many new sequences, the DVC is barely visible on high resolution MRI and is not visible on planning CT scans. In a previous study, Williams et al. described the visualization of enhancement in the area postrema in a case report by using MR imaging (19). However, Horsburgh et al. have recently studied the normal appearance and contrast enhancement of CVO on routine clinical brain MRI images. The AP was enhanced in only $2 \%$ of their cases (9). Therefore, the present atlas study was carried out using microscopic and endoscopic descriptions of the DVC (10-16). We have determined boundaries for different organs implicated in RINV with easy anatomic landmarks. In order to test the reproducibility of the DVC delineation, we have performed two interobserver analyses. The eight radiation oncologists of the working group have delineated the DVC, for the first time, by using a first tutorial as model. The DVC on this tutorial was very similar to the real DVC, particularly regarding the small size and volume $\left(0.4 \mathrm{~cm}\right.$ and $0.04 \mathrm{~cm}^{3}$, respectively). Nonetheless, we obtained poor reproducibility $(\mathrm{KI}=0.4)$. Then, following discussions, we decided to slightly expand the volume of the structure (by using a circle of $4 \mathrm{~mm}$ in diameter) and to accurately define the caudal limit (the transversal plane of the obex). The second interobserver analysis showed better results $(\mathrm{KI}=0.70)$ and validated the second tutorial as a practical contouring guideline to be used for an MR-based atlas for the delineation of the DVC. The three parts of the brainstem have rarely been delimited, so this study was also an opportunity to find a consensus on this subject.

The strength of our study is that it is multicentric and multidisciplinary. Indeed, the ten radiation oncologists came from seven different French departments of Radiation Oncology and the atlas was validated by a neuroanatomopathologist (HMD), a neurosurgeon (ML), a neuroanatomist, and neuroradiologist $(\mathrm{DH})$. Nevertheless, this contouring atlas is based on MRI so limits the cases to those for which MR is available. Finally, this RINV atlas may be used in routine practice and will have to be further evaluated in different clinical situations using different irradiation techniques. The atlas may be used in prospective studies and in routine use to share better knowledge of these organs with the aim of improving the quality of life of patients after irradiation for head and neck cancer. 
Conflicts of interest: None declared

Acknowledgments: The authors acknowledge AQUILAB SAS for their contribution to this study.

\section{References}

1. Nutting CM, Morden JP, Harrington KJ, Urbano TG, Bhide SA, Clark C, et al. Parotid-sparing intensity modulated versus conventional radiotherapy in head and neck cancer (PARSPORT): a phase 3 multicentre randomised controlled trial. Lancet Oncol. 2011;12(2):127-36.

2. Rosenthal DI, Chambers MS, Fuller CD, Rebueno NC, Garcia J, Kies MS, et al. Beam path toxicities to non-target structures during intensity-modulated radiation therapy for head and neck cancer. Int J Radiat Oncol Biol Phys. 2008;72(3):747-55.

4. Miller AD, Leslie RA. The area postrema and vomiting. Front Neuroendocrinol. 1994;15(4):301-20.

5. Monroe AT, Reddy SC, Gibbs GL, White GA, Peddada AV. Factors associated with radiationinduced nausea and vomiting in head and neck cancer patients treated with intensity modulated radiation therapy. Radiother Oncol. 2008;87(2):188-94.

6. Wang TJC, Fontenla S, McCann P, Young RJ, McNamara S, Rao S, et al. Correlation of Planned Dose to Area Postrema and Dorsal Vagal Complex 3. Alexander E, 3rd, Siddon RL, Loeffler JS. The acute onset of nausea and vomiting following stereotactic radiosurgery: correlation with total dose to area postrema. Surgical neurology. 1989;32(1):40-4.

with Clinical Symptoms of Nausea and Vomiting in Oropharyngeal Cancer (OPC) patients treated with radiation alone using IMRT. J Radiat Oncol. 2013;2(4):407-12.

7. Ciura K, McBurney M, Nguyen B, Pham M, Rebueno N, Fuller CD, et al. Effect of brain stem and dorsal vagus complex dosimetry on nausea and vomiting in head and neck intensity-modulated radiation therapy. Med Dosim. 2011;36(1):41-5.

8. Kocak-Uzel E, Gunn GB, Colen RR, Kantor ME, Mohamed AS, Schoultz-Henley S, et al. Beam path toxicity in candidate organs-at-risk: assessment of radiation emetogenesis for patients receiving head and neck intensity modulated radiotherapy. Radiother Oncol. 2014;111(2):281-8.

9. Horsburgh A, Massoud TF. The circumventricular organs of the brain: conspicuity on clinical 3T MRI and a review of functional anatomy. Surg Radiol Anat. 2013;35(4):343-9.

10. Duvernoy HM, Koritké, J. G., Monnier G., Jacquet G. Sur la vascularisation de l'area postrema et de la face postérieure du bulbe chez l'homme. Z Anat Entwickl-Gesh. 1972;138:1972.

11. Duvernoy HM. Human Brainstem vessels second edition. Springer Verlag Berlin Heidelberg. 1999.

12. Duvernoy HM, Risold PY. The circumventricular organs: an atlas of comparative anatomy and vascularization. Brain Res Rev. 2007;56(1):119-47.

13. Naidich T. P. DHM, Delman B. N., Sorensen A. G., Kolliors S. and Haacke B.N. Duvernoy's atlas of the Human Brain Stem and Cerebellum. Springer Verlag. 2009.

14. Longatti $P$, Porzionato A, Basaldella L, Fiorindi A, De Caro P, Feletti $A$. The human area postrema: clear-cut silhouette and variations shown in vivo. J Neurosurg. 2015;122(5):989-95.

15. Angeles Fernandez-Gil M, Palacios-Bote R, Leo-Barahona M, Mora-Encinas JP. Anatomy of the brainstem: a gaze into the stem of life. Semin Ultrasound CT MR. 2010;31(3):196-219.

16. Paxinos G, Huang, X., Sengul, G.,Watson, C. Organization of brainstem nuclei. In: Press AEA, editor. The Human Nervous System 2012. p. 260-327.

17. Dewas S, Bibault JE, Blanchard P, Vautravers-Dewas C, Pointreau Y, Denis F, et al. Delineation in thoracic oncology: a prospective study of the effect of training on contour variability and dosimetric consequences. Radiat Oncol. 2011;6:118.

18. Lee VH, Ng SC, Leung TW, Au GK, Kwong DL. Dosimetric predictors of radiation-induced acute nausea and vomiting in IMRT for nasopharyngeal cancer. Int $\mathrm{J}$ Radiat Oncol Biol Phys. 2012;84(1):176-82.

19. Williams KD, Dean B, Drayer BP. Demonstration of the area postrema with contrast-enhanced MR. AJNR Am J Neuroradiol. 1990;11(4):733-4. 

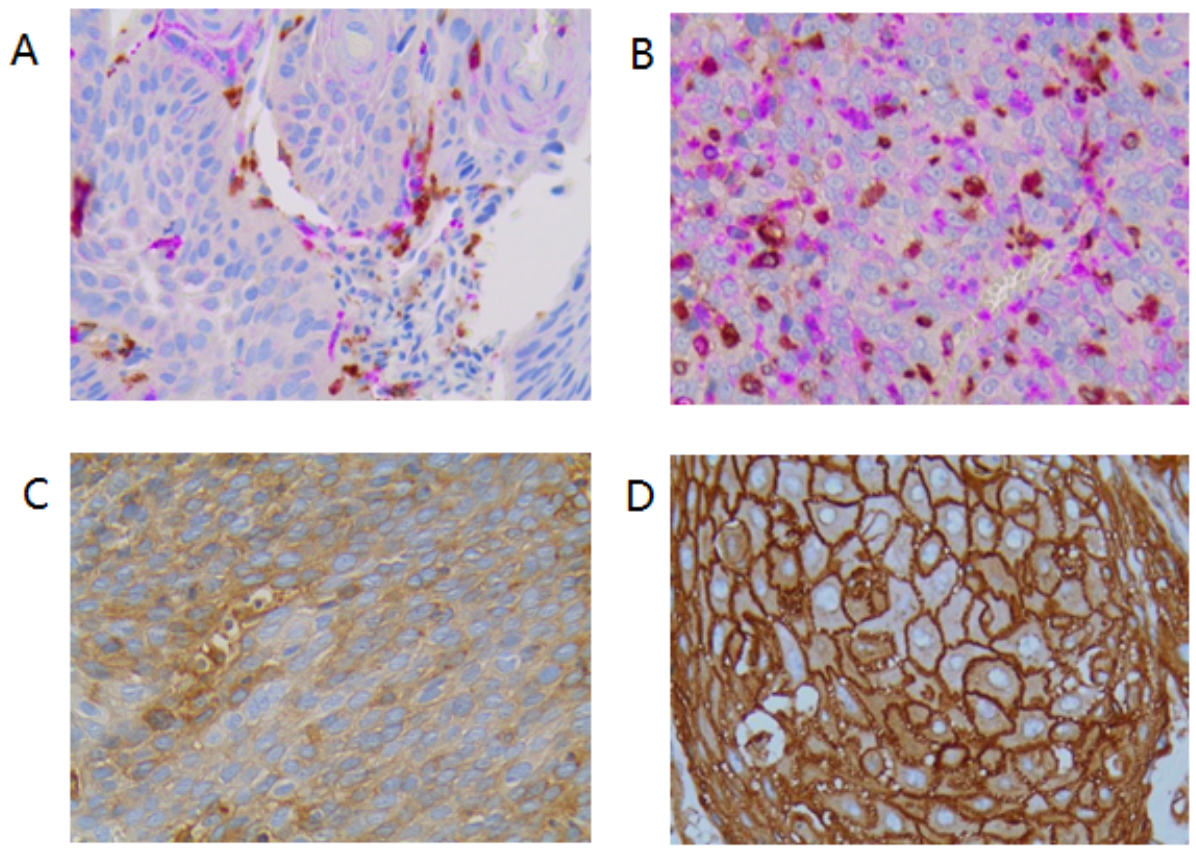

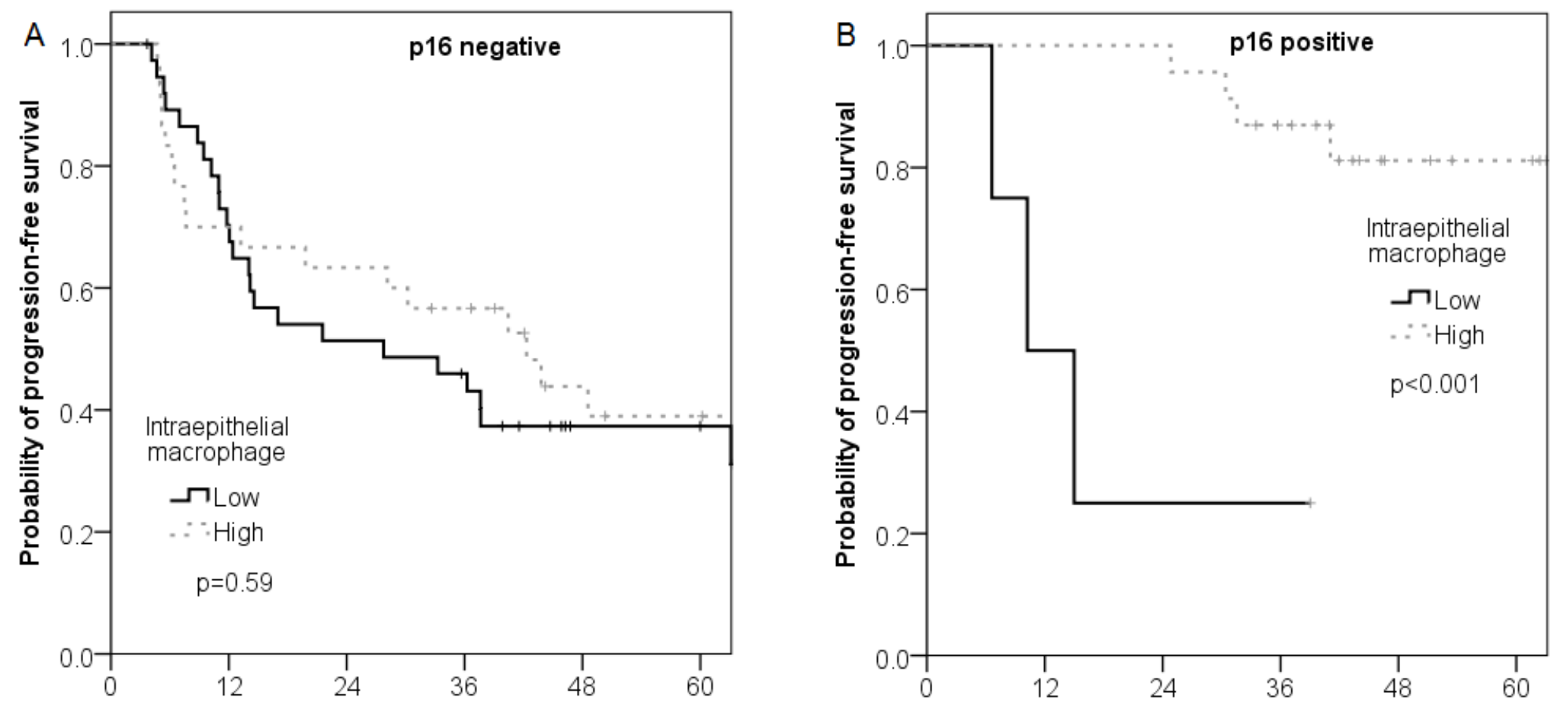

No. at risk

Time (months)

\begin{tabular}{cccccc} 
No. at risk & \multicolumn{5}{c}{ Time (months) } \\
-4 & 2 & 1 & 1 & 0 & 0 \\
----23 & 23 & 23 & 18 & 9 & 7
\end{tabular}

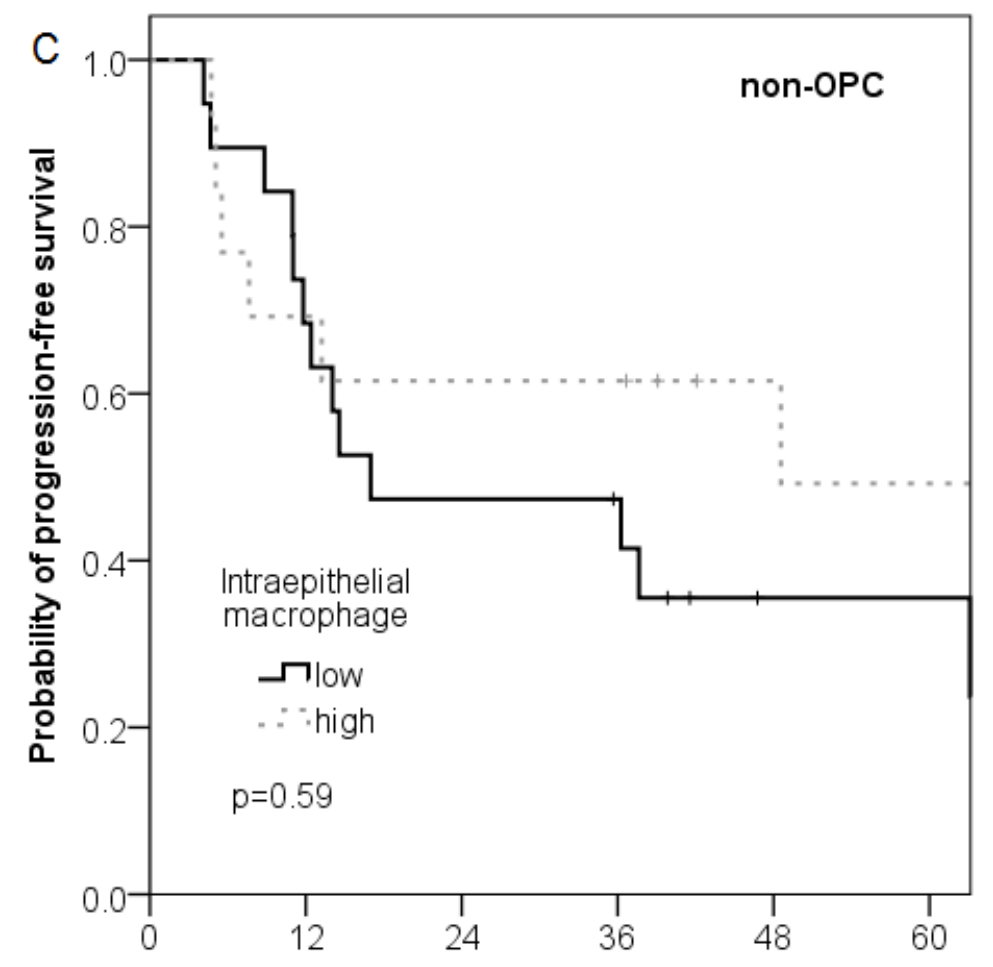

No. at risk

$\begin{array}{lllll}26 & 19 & 16 & 7 & 6 \\ 21 & 19 & 16 & 9 & 7\end{array}$

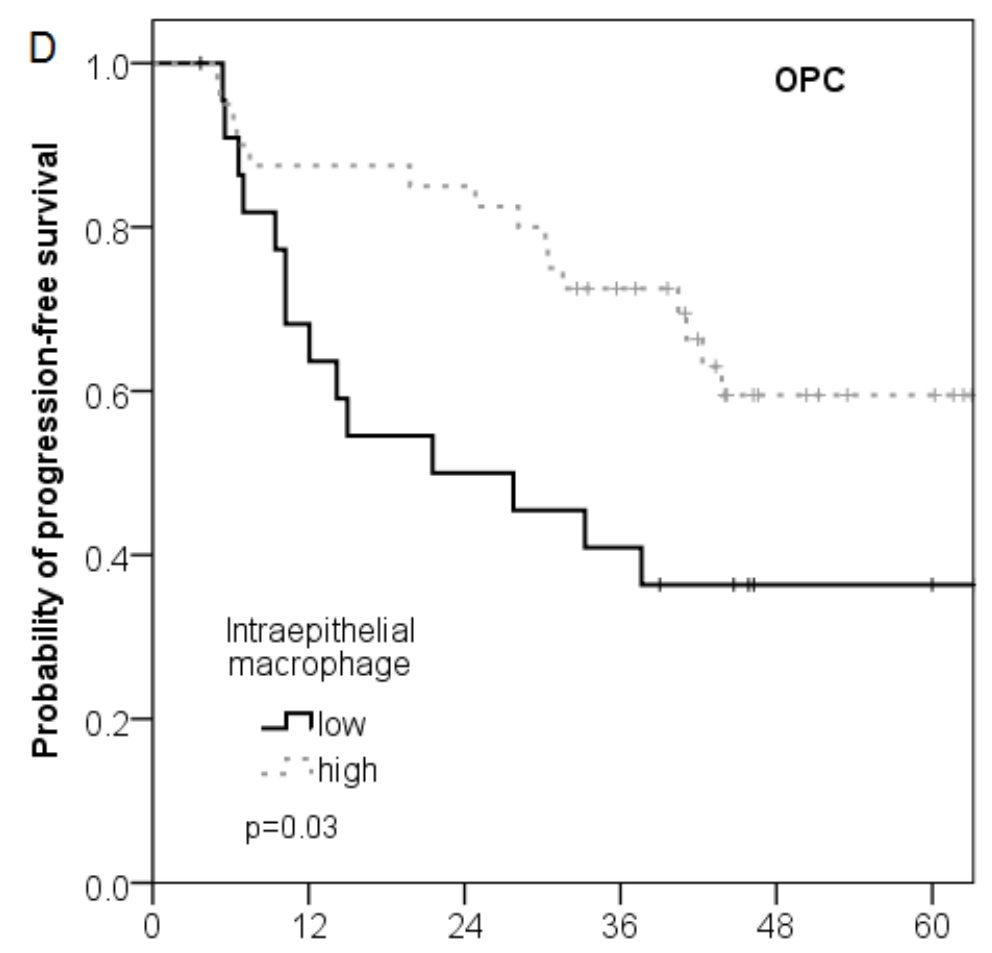

No. at risk Time (months)

$\begin{array}{rrrrrrrrrrrr}19 & 13 & 9 & 8 & 3 & 3 & -23 & 15 & 11 & 9 & 4 & 3 \\ 13 & 9 & 8 & 8 & 5 & 4 & ----40 & 35 & 34 & 26 & 13 & 10\end{array}$


Low M2 in stroma

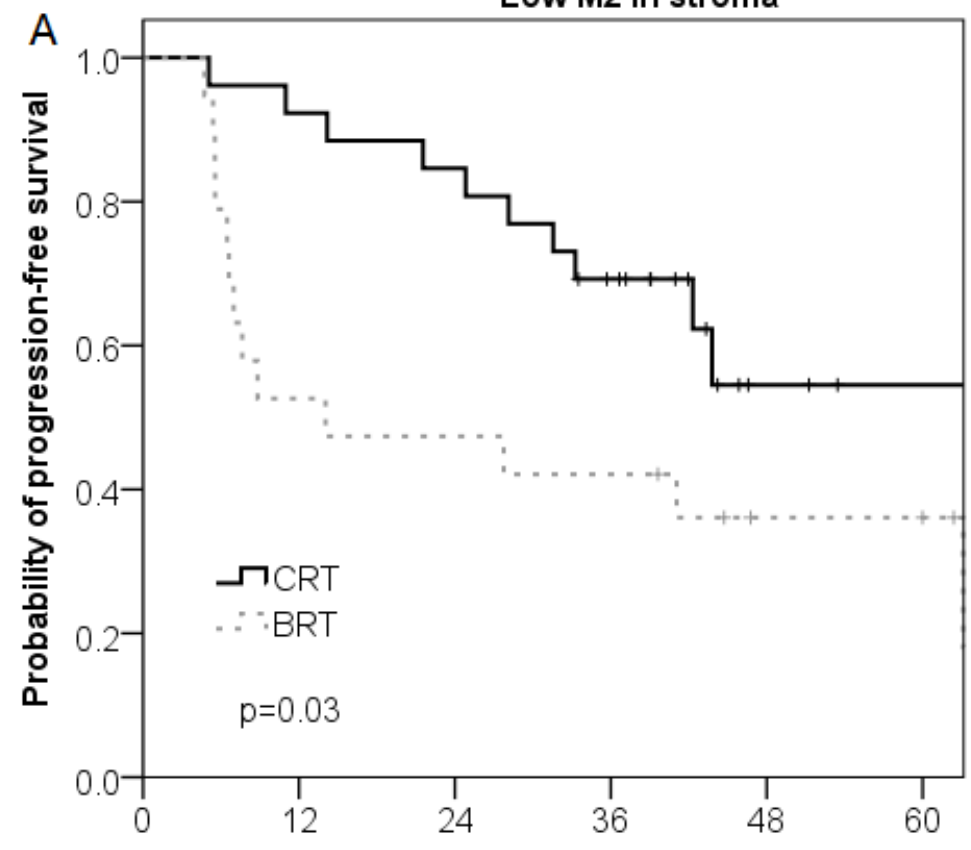

No. at risk

CRT 26

BRT 19
24

Time (months)

$10 \quad 9$

16

8
4

4

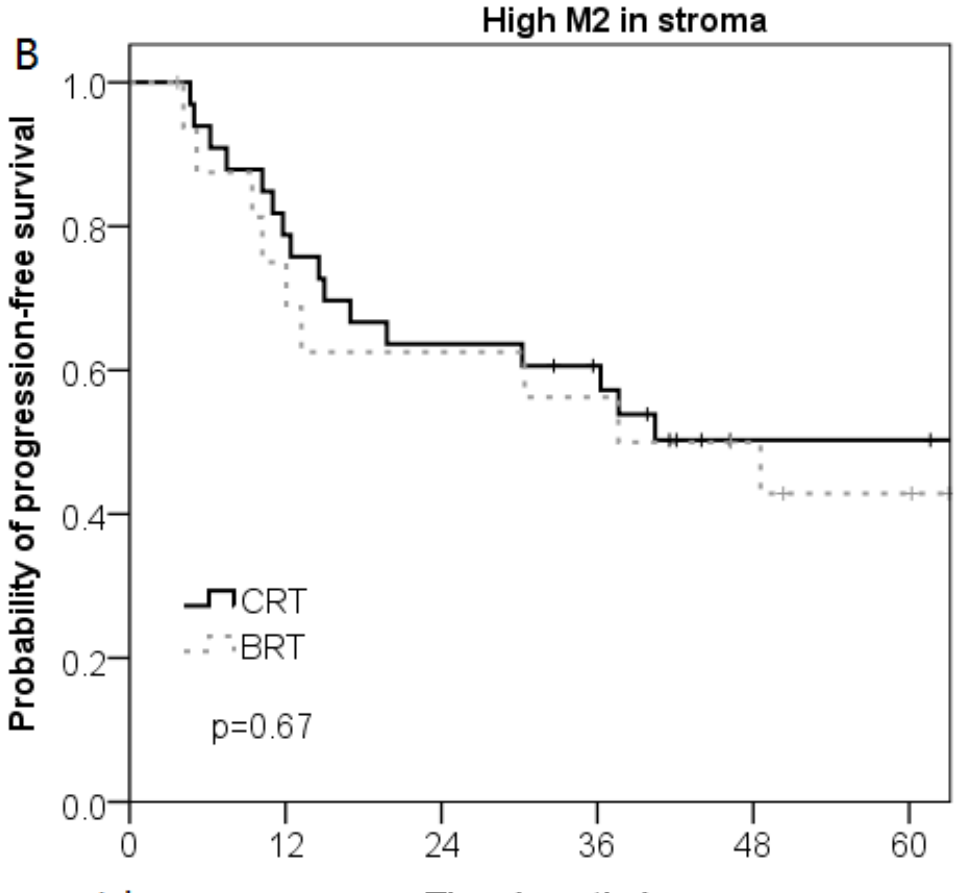

No. at risk

CRT 33

26

21

18

BRT 17

12

10

9

10

10

5 
Table 1. Correlation of expression of biomarkers with clinicopathological parameters in the whole population $(N=95)$

\begin{tabular}{|c|c|c|c|c|c|c|c|c|c|c|c|c|c|c|c|c|}
\hline \multirow[t]{2}{*}{ Characteristics } & \multirow[t]{2}{*}{$N(\%)$} & \multicolumn{3}{|c|}{ IEMD } & \multicolumn{3}{|c|}{$\% \mathrm{M}$ in stroma } & \multicolumn{3}{|c|}{$\mathrm{M} 2 / \mathrm{M}$ in stroma } & \multicolumn{3}{|c|}{$\% \mathrm{M} 2$ in stroma } & \multicolumn{3}{|c|}{ HLA class I } \\
\hline & & low & high & $p$ & low & high & $p$ & low & high & $\mathrm{p}$ & low & high & $\mathrm{p}$ & Low & High & $p$ \\
\hline Age (years) & & & & 0.72 & & & 0.50 & & & 0.68 & & & 0.98 & & & 0.07 \\
\hline$<65$ & $74(78)$ & 32 & 42 & & 27 & 47 & & 28 & 46 & & 35 & 39 & & 33 & 41 & \\
\hline$>=65$ & $21(22)$ & 10 & 11 & & 6 & 15 & & 9 & 12 & & 10 & 11 & & 14 & 7 & \\
\hline Gender & & & & 0.47 & & & 0.45 & & & 0.75 & & & 1.0 & & & 0.84 \\
\hline Male & $76(80)$ & 35 & 41 & & 25 & 51 & & 29 & 47 & & 36 & 40 & & 38 & 38 & \\
\hline Female & $19(20)$ & 7 & 12 & & 8 & 11 & & 8 & 11 & & 9 & 10 & & 9 & 10 & \\
\hline Smoking status & & & & 0.31 & & & 0.14 & & & 0.63 & & & 0.89 & & & 0.88 \\
\hline Never/Former & $64(67)$ & 26 & 38 & & 19 & 45 & & 26 & 38 & & 30 & 34 & & 32 & 32 & \\
\hline Current & $31(33)$ & 16 & 15 & & 14 & 17 & & 11 & 20 & & 15 & 16 & & 15 & 16 & \\
\hline PS (ECOG) & & & & 0.98 & & & 0.04 & & & 0.55 & & & 0.14 & & & 0.77 \\
\hline 0 & $70(74)$ & 31 & 39 & & 20 & 50 & & 26 & 44 & & 30 & 40 & & 34 & 36 & \\
\hline$\geq 1$ & $25(26)$ & 11 & 14 & & 13 & 12 & & 11 & 14 & & 15 & 10 & & 13 & 12 & \\
\hline Charlson index & & & & 0.06 & & & 0.42 & & & 0.63 & & & 0.89 & & & 0.56 \\
\hline $0-1$ & $64(67)$ & 24 & 40 & & 24 & 40 & & 26 & 38 & & 30 & 34 & & 33 & 31 & \\
\hline$\geq 2$ & $31(33)$ & 18 & 13 & & 9 & 22 & & 11 & 20 & & 15 & 16 & & 14 & 17 & \\
\hline Location & & & & $\mathbf{0 . 0 3}$ & & & 0.96 & & & 0.52 & & & 0.35 & & & 0.94 \\
\hline Oropharynx & $63(66)$ & 23 & 40 & & 22 & 41 & & 26 & 37 & & 32 & 31 & & 31 & 32 & \\
\hline Non-oropharynx & $32(34)$ & 19 & 13 & & 11 & 21 & & 11 & 21 & & 13 & 19 & & 16 & 16 & \\
\hline $\mathrm{T}$ classification & & & & 0.06 & & & 0.16 & & & 0.11 & & & 0.60 & & & 0.09 \\
\hline $\mathrm{T} 1-3$ & $74(78)$ & 29 & 45 & & 23 & 51 & & 32 & 42 & & 34 & 40 & & 40 & 34 & \\
\hline $\mathrm{T} 4$ & $21(22)$ & 13 & 8 & & 10 & 11 & & 5 & 16 & & 11 & 10 & & 7 & 14 & \\
\hline $\mathrm{N}$ classification & & & & 0.04 & & & 0.15 & & & 0.14 & & & 0.55 & & & 0.60 \\
\hline N0-1 & $45(47)$ & 25 & 20 & & 19 & 26 & & 14 & 31 & & 23 & 22 & & 21 & 24 & \\
\hline $\mathrm{N} 2-3$ & $50(53)$ & 17 & 33 & & 14 & 36 & & 23 & 27 & & 22 & 27 & & 26 & 24 & \\
\hline Overall stage & & & & 0.68 & & & 0.33 & & & 0.59 & & & 0.70 & & & 0.61 \\
\hline III & $34(36)$ & 16 & 18 & & 14 & 20 & & 12 & 22 & & 17 & 17 & & 18 & 16 & \\
\hline IV & $61(64)$ & 26 & 35 & & 19 & 42 & & 25 & 36 & & 28 & 33 & & 29 & 32 & \\
\hline HPV/p16 status & & & & $<0.001$ & & & 0.86 & & & 0.002 & & & 0.06 & & & 0.003 \\
\hline p16+ & $27(28)$ & 4 & 23 & & 9 & 18 & & 17 & 10 & & 17 & 10 & & 20 & 7 & \\
\hline P16- & $68(72)$ & 38 & 30 & & 24 & 44 & & 20 & 48 & & 28 & 40 & & 27 & 41 & \\
\hline Concurrent regimen & & & & 0.09 & & & 0.51 & & & 0.66 & & & 0.41 & & & 0.08 \\
\hline CRT & $59(62)$ & 22 & 37 & & 19 & 40 & & 24 & 35 & & 26 & 33 & & 25 & 34 & \\
\hline BRT & $36(38)$ & 20 & 16 & & 14 & 22 & & 13 & 23 & & 19 & 17 & & 22 & 14 & \\
\hline
\end{tabular}




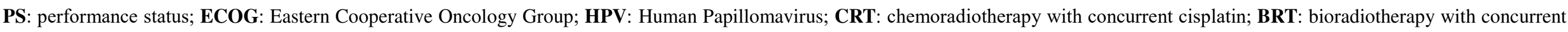
cetuximab; IEMD: intraepithelial macrophage density 
Table 2. Correlation between biomarkers

\begin{tabular}{llll}
\hline & Intraepithelial $\mathrm{M}_{\mathrm{s}}(p)$ & $\% \mathrm{M}$ in stroma $\mathrm{r}_{\mathrm{s}}(p)$ & $\% \mathrm{M} 2$ in stroma $\mathrm{r}_{\mathrm{s}}(p)$ \\
\hline Intraepithelial $\mathrm{M}$ & $/$ & $/$ & $/$ \\
$\% \mathrm{M}$ in stroma & $0.21(0.04)$ & $/$ & $/$ \\
$\% \mathrm{M} 2$ in stroma & $0.06(0.55)$ & $0.05(0.65)$ & $/$ \\
$\mathrm{HLA}$ class I & $-0.03(0.75)$ & $-0.14(0.18)$ & $0.12(0.25)$ \\
\hline
\end{tabular}


Table 3. Univariate and multivariate analyses of overall survival (OS) and progression-free survival (PFS) of the entire population (N=95).

Characteristics OS

\begin{tabular}{|c|c|c|c|c|c|c|c|c|}
\hline \multirow[t]{3}{*}{ Characteristics } & \multicolumn{4}{|c|}{ OS } & \multicolumn{4}{|c|}{ PFS } \\
\hline & \multicolumn{2}{|c|}{ UVA } & MVA & \multirow[b]{2}{*}{$\mathrm{P}$} & \multicolumn{2}{|c|}{ UVA } & MVA & \multirow[b]{2}{*}{$\mathrm{P}$} \\
\hline & HR & $P$ & $\begin{array}{l}\mathrm{HR} \\
\mathrm{CI})\end{array}$ & & HR & $\mathrm{P}$ & $\mathrm{HR}(95 \% \mathrm{CI})$ & \\
\hline $\begin{array}{l}\text { T4 classification } \\
\text { (T1-3) }\end{array}$ & 3.3 & $<0.001$ & $2.6(1.2-6.0)$ & 0.02 & 3.8 & $<0.001$ & $3.2(1.6-6.4)$ & 0.001 \\
\hline $\begin{array}{l}\text { N2-3 classification } \\
\text { (N0-1) }\end{array}$ & 1.1 & 0.73 & $2.1(0.9-4.7)$ & 0.07 & 1.3 & 0.35 & $1.8(0.9-3.6)$ & 0.09 \\
\hline $\begin{array}{l}\text { Never/Former smoker } \\
\text { (Current) }\end{array}$ & 0.6 & 0.08 & $0.4(0.2-0.8)$ & 0.007 & 0.6 & 0.08 & $0.4(0.2-0.8)$ & 0.01 \\
\hline $\begin{array}{l}\text { BRT } \\
\text { (CRT) }\end{array}$ & 1.7 & 0.11 & $1.7(0.9-3.5)$ & 0.11 & 1.7 & 0.06 & $1.9(1.0-3.5)$ & 0.049 \\
\hline $\begin{array}{l}\text { Teatment duration } \geqslant 50 \text { days } \\
(<50 \text { days })\end{array}$ & 1.8 & 0.05 & $3.5(1.7-7.2)$ & $<0.001$ & 1.6 & 0.08 & $2.6(1.4-4.9)$ & 0.004 \\
\hline $\begin{array}{l}\mathrm{PS}=0 \\
(\mathrm{PS} \geq 1)\end{array}$ & 0.5 & 0.046 & $0.6(0.3-1.2)$ & 0.15 & 0.4 & 0.001 & $0.5(0.2-0.99)$ & 0.046 \\
\hline $\begin{array}{l}\text { p16+ } \\
\text { (p16-) }\end{array}$ & 0.2 & 0.003 & $0.3(0.1-0.9)$ & 0.04 & 0.4 & 0.01 & $0.6(0.1-1.4)$ & 0.21 \\
\hline $\begin{array}{l}\mathrm{M} \text { in stroma low } \\
\text { (high) }\end{array}$ & 1.6 & 0.15 & $1.3(0.6-2.8)$ & 0.43 & 1.2 & 0.45 & & \\
\hline $\begin{array}{l}\text { M2/M in stroma low } \\
\text { (high) }\end{array}$ & 0.7 & 0.36 & & & 0.9 & 0.67 & & \\
\hline $\begin{array}{l}\text { M2 in stroma low } \\
\text { (high) }\end{array}$ & 1.0 & 0.90 & & & 1.0 & 0.99 & & \\
\hline $\begin{array}{l}\text { Intraepithelial M low } \\
\text { (high) }\end{array}$ & 2.0 & 0.03 & $2.2(0.98-4.8)$ & 0.06 & 2.0 & 0.01 & $2.0(1.02-4.1)$ & 0.04 \\
\hline $\begin{array}{l}\text { HLA-I low } \\
\text { (high) }\end{array}$ & 0.5 & 0.07 & $0.6(0.3-1.2)$ & 0.17 & 0.7 & 0.17 & $0.8(0.4-1.5)$ & 0.46 \\
\hline
\end{tabular}

CRT: chemoradiotherapy with concurrent cisplatin; BRT: bioradiotherapy with concurrent cetuximab; PS: performance status 